\title{
Aberrant methylation of the CADM1 promoter is associated with poor prognosis in hepatocellular carcinoma treated with liver transplantation
}

\author{
WU ZHANG ${ }^{1}$, LIN ZHOU ${ }^{1}$, SONG-MING DING ${ }^{1}$ HAI-YANG XIE ${ }^{1}$, XIAO XU ${ }^{2}$, JIAN WU ${ }^{2}$, \\ QI-XIN CHEN ${ }^{1}$, FENG ZHANG $^{1}$, BA-JING WEI ${ }^{1}$, AHMED TAKI ELDIN $^{1}$ and SHU-SEN ZHENG ${ }^{1,2}$ \\ ${ }^{1}$ Key Laboratory of Combined Multi-organ Transplantation, Ministry of Public Health; ${ }^{2}$ Division of Hepatobiliary \\ and Pancreatic Surgery, First Affiliated Hospital, School of Medicine, Zhejiang University, Hangzhou, P.R. China
}

Received November 23, 2010; Accepted December 29, 2010

DOI: $10.3892 /$ or.2011.1159

\begin{abstract}
Approximately 20-40\% of hepatocellular carcinoma (HCC) patients who undergo liver transplantation (LT) experience HCC recurrence within 5 years of the operation. Current predictors cannot sufficiently differentiate patients at risk for biochemical recurrence. The aim of the present study was to investigate the methylation status and expression levels of cell adhesion molecule 1 (CADM1) in HCC; to elucidate its regulation mechanisms; and finally, to evaluate the potential predictive value for tumor recurrence. Aberrant hypermethylation of CADM1 was frequently found in HCC cell lines with decreased CADM1 mRNA by bisulfite sequencing PCR. Re-expression of CADM1 was induced by treatment with demethylating agents. The promoter region of CADM1 was identified and the basal promoter activity was located in the -226 to -146 region relative to the transcriptional start site (TSS). Site-directed mutagenesis revealed that the consensus Sp1 binding site located in the basal promoter region was important for mediating CADM1 promoter activity. Furthermore, aberrant hypermethylation of CADM1 was detected in 34 of 82 (41.5\%) of HCC tissues. The recurrence rate of the patients with CADM1 methylation was higher compared to that without CADM1 methylation (70.6\% versus 33.3\%; $\mathrm{P}=0.001)$. Multivariate analysis revealed that CADM1 methylation status (HR $=2.788$; 95\% CI, 1.043-5.063; $\mathrm{P}=0.010$ ) was an independent prognostic factor for disease-free survival (DFS) of HCC patients treated with LT. In conclusion, CADM1 methylation may be used as a potential predictive biomarker for tumor recurrence of HCC after LT.
\end{abstract}

Correspondence to: Professor Shu-Sen Zheng, Key Laboratory of Combined Multi-organ Transplantation, Ministry of Public Health; Division of Hepatobiliary and Pancreatic Surgery, First Affiliated Hospital, School of Medicine, Zhejiang University, Hangzhou, P.R. China

E-mail: shusenzheng@zju.edu.cn

Key words: cell adhesion molecule 1, DNA methylation, prognosis, hepatocellular carcinoma, liver transplantation

\section{Introduction}

Hepatocellular carcinoma (HCC) is one of the most common malignancies in the world and annually accounts for up to 1 million deaths worldwide (1). Etiological factors for HCC are well-defined, heavy alcohol intake, aflatoxin intake and infection with hepatitis $\mathrm{B}$ or $\mathrm{C}$ virus are important risk factors for HCC. However, the detailed molecular mechanisms for the development and progression of HCC remain unclear (2). Liver transplantation (LT) offers a potential curative choice for patients with early HCC. But, recurrence of HCC following LT remains one of the most prevalent causes leading to poor long-term survival (3). Therefore, further studies focus on identifying significant predictors of recurrence in HCC patients after LT are highlighted accordingly.

Epigenetic modification has been identified as a crucial event in carcinogenesis (4). Remarkably, neoplastic-related DNA methylation leading to inactivation of tumor suppressor genes (TSG) is thought to be an early mechanism of human carcinogenesis (5). Hypermethylation of the $\mathrm{CpG}$ island in promoter region can play important roles to affect genes involved in the cell growth, apoptosis, cell-to-cell interaction, DNA repair and angiogenesis, all of which are involved in the development and progression of cancer (6). Over recent years aberrant DNA methylation was shown to be a promising biomarker for monitoring prognosis of patients with cancer $(7,8)$.

Cell adhesion molecule 1 (CADM1), was recently identified as a novel tumor suppressor gene in human non-small cell lung cancer (NSCLC) through a series of functional complementation analysis (9). Loss of CADM1 expression are frequently observed in various cancers, including NSCLC, nasal NK/T-cell lymphoma, colorectal carcinoma and cervical cancer, especially in those with invasion or metastasis (9-12). DNA methylation has been shown to be the major mechanism for CADM1 inactivation in different types of tumors (13). Heller et al (14) revealed that methylation of CADM1, leading to loss of its expression, is an important event in the tumorigenesis of NSCLC. Lung et al (15) found that CADM1 was associated with metastasis in nasopharyngeal carcinoma. In the present study, we identified the proximal promoter region of CADM1 gene for the first time. Moreover, the correlation of 
CADM1 promoter methylation with clinicopathologic features was determined in HCC patients treated with LT.

\section{Materials and methods}

Cells lines and tissue samples. A total of 7 liver cell lines (SMMC-7721, HepG2, Hep3B, SK-HEP-1, MHCC-97L, MHCC-97H and MHCC-LM3) were examined in this study. Two immortalized normal liver cell lines (Chang Liver and L02) were used as normal control for HCC. Eighty-two HCC patients (75 male, 7 female; mean age 49.2 years; range 22-67 years) who underwent LT in our center during 2004 to 2007 were enrolled in this retrospective study according to the eligibility criteria used in our previous study (16). Normal liver tissues $(n=8)$ were obtained from liver donors during living donor liver transplantation as controls. Diagnoses were confirmed by histopathological examination. Liver tissues were frozen at immediately in liquid nitrogen after resection, and stored at $-80^{\circ} \mathrm{C}$. Written informed consent was obtained from each patient, and the study was approved by the local ethics committee according to the Declaration of Helsinki.

RNA extraction and real-time quantitative reverse transcription-PCR. Total RNA was isolated from all cell lines using TRIzol reagent (Invitrogen). First strand cDNA was generated using the SuperScript III first-strand synthesis system (Invitrogen). Quantitative reverse transcription-PCR (qRT-PCR) was performed using an ABI Prism $7500^{\mathrm{TM}}$ instrument (Applied Biosystems). The CADM1-specific primers are shown in Table I. GAPDH was used as internal control. Relative expression levels of CADM1 were calculated according to the $2^{-\Delta \Delta C T}$ method (17).

5-Aza-2'-deoxycytidine treatment. To detect reactivation of CADM1 expression, HCC cell lines were treated with a DNA methyltransferase inhibitor 5-Aza-2'-deoxycytidine (5-Aza-dC; Sigma) at a final concentration of $10 \mu \mathrm{M}$ for 3 days. Fresh medium containing 5-Aza-dC was changed every $24 \mathrm{~h}$. Total RNA was extracted as described previously.

DNA extraction and bisulfite sequencing PCR (BSP). Genomic DNA was extracted from the cell lines and frozen liver tissues using QIAamp DNA mini kit (Qiagen). DNA samples $(1 \mu \mathrm{g})$ were chemically modified with EpiTect Bisulfite Kit (Qiagen) according to the manufacturer's instructions. The CADM1 putative promoter region was predicted by Genomatix Gene2Promoter software (http://www.genomatix. de/) to be from -500 to +211 relative to the TSS. Sodium bisulfite-treated DNA was amplified by HotStar Taq DNA Polymerase kit (Qiagen) for region (-429 to +39) of CADM1 gene. The primers are shown in Table I. PCR conditions were as follows: 1 cycle at $95^{\circ} \mathrm{C}$ for 5 min followed by 40 cycles of $95^{\circ} \mathrm{C}$ for $30 \mathrm{sec}, 54^{\circ} \mathrm{C}$ for $30 \mathrm{sec}, 72^{\circ} \mathrm{C}$ for $30 \mathrm{sec}$, and a final extension at $72^{\circ} \mathrm{C}$ for $10 \mathrm{~min}$. The PCR products were then cloned into the pGEM T-easy vector (Promega), with eight colonies randomly selected and sequenced.

Generation of luciferase-reporter constructs. A 1833-bp fragment spanning from -1599 to +234 of the CADM1 promoter region was prepared by PCR amplification of the human genomic DNA using a sense primer $-1599 \mathrm{~F}$ containing a $\mathrm{KpnI}$ restriction site and an antisense primer $+234 \mathrm{R}$ containing an $X h o I$ restriction site. The purified PCR products then cloned into the pGEM T-easy vector and recloned into the KpnI and $\mathrm{XhoI}$ sites in the luciferase reporter vector pGL 4.17 (Promega). Using this construct as the parent, PCR-mediated deletions were performed. The PCR reactions contained a similar antisense primer $+234 \mathrm{R}$ and one of the sense primers (information of primers shown in Table I). All constructs were sequenced in both directions to ensure correct nucleotide sequence. The normal liver cell line (Chang Liver) was co-transfected with CADM1 promoter constructs and Renilla luciferase vector as an internal control, using lipofectamine 2000 kit (Invitrogen) according to the manufacturer's instructions. The luciferase reporter gene activities were measured using Dual-Luciferase Reporter Assay System (Promega) as specified by the manufacturer. Each experiment was done in triplicate and repeated at least three times.

Site-directed mutagenesis. Based on the findings of luciferase reporter assay, mutations in the putative $\mathrm{Sp1}$ binding sites were created using a Quick-Change Lightning multi site-directed mutagenesis kit (Stratagene) according to the manufacturer's instructions. Using the luciferase-reporter construct (-226 to +234 ) as a template, three-bases change was introduced at a location -206 to -208 relative to the TSS (denoted as -206 C-A, $-207 \mathrm{G}-\mathrm{A},-208 \mathrm{C}-\mathrm{T}$ ). The three bases change was predicted by the transcription element search system (TESS, University of Pennsylvania) to abolish the binding site for Sp1 transcription factor. Primers for mutagenesis of Sp1 binding site are shown in Table I. Mutant construct was sequenced, and the exact sequences are shown in the relevant figure. Luciferase reporter assay was performed as described previously.

Loss of heterozygosity $(\mathrm{LOH})$ analysis. $\mathrm{LOH}$ analysis in 32 paired HCC tissues was determined based upon an analysis of three microsatellite markers (centromeric, D11S1885; intragenic, D11S908; telomeric, D11S1992). DNA was amplified by multiplex PCR with fluorescent-labeled oligonucleotides flanking the microsatellite markers. The information of primers in detail is shown in Table I. PCR conditions were as follows: $15 \mathrm{~min}$ of initial denaturation at $95^{\circ} \mathrm{C}$, followed by an 11-touch-down cycles consisting of $94^{\circ} \mathrm{C}$ for $20 \mathrm{sec}, 62^{\circ} \mathrm{C}$ for $40 \mathrm{sec}$ (decrease $0.5^{\circ} \mathrm{C}$ per cycle), $68^{\circ} \mathrm{C}$ for $2 \mathrm{~min}$, then 24 cycles of $94^{\circ} \mathrm{C}$ for $20 \mathrm{sec}, 56^{\circ} \mathrm{C}$ for $40 \mathrm{sec}, 68^{\circ} \mathrm{C}$ for $2 \mathrm{~min}$, with a final extension at $60^{\circ} \mathrm{C}$ for $1 \mathrm{~h}$. The PCR products were separated by capillary electrophoresis on an ABI 3130 Genetic Analyzer (Applied Biosystems). The data were collected and processed using Genescan and GeneMapper 4.0 software (Applied Biosystems). LOH index of $<0.6$ or $>1.7$ were considered positive as described previously (18). Each LOH-positive case was repeated at least twice.

Construction of the expression vector for CADM1 and Western blotting identification. Full-length sequence of CADM1 cDNA was amplified by PCR with a panel of human lymph cDNA (Clontech) as a template. Primers were designed with XhoI and $K p n I$ restriction sites in them, respectively (sequence information of primers shown in Table I). Amplified fulllength fragment was digested with $\mathrm{XhoI} / \mathrm{KpnI}$ and ligated 
o o o o n n o o o o o o o 光 จิ

$\sum$

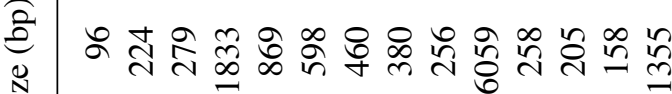
is
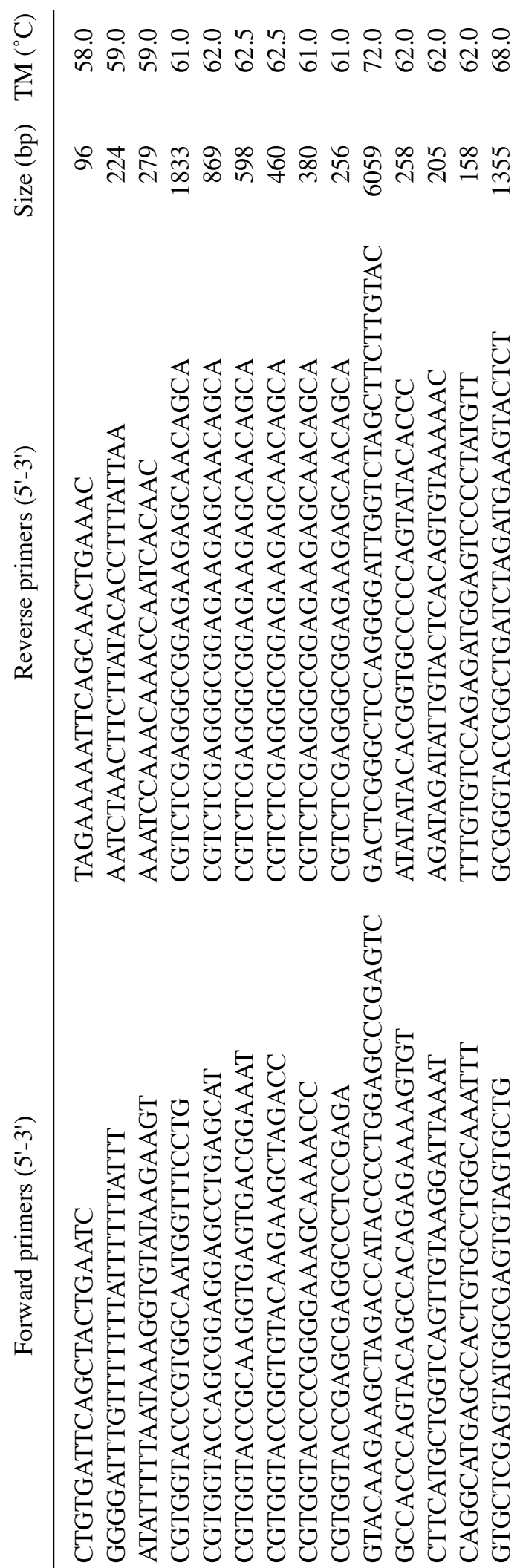

है

$1+$

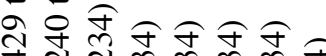

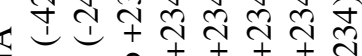

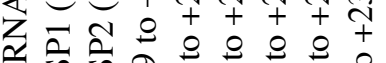

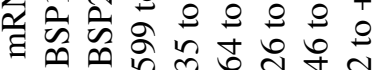
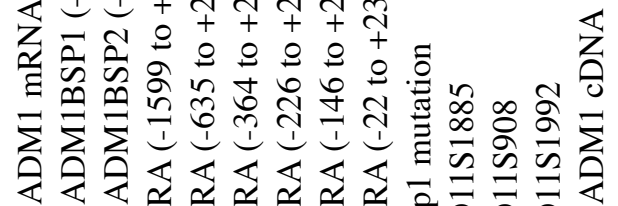

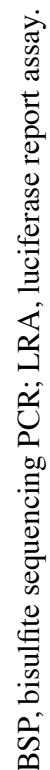

into pcDNA3.1 (-) vector. The sequence of the recombinant plasmid was verified by sequencing of both strands. To detect ectopic expression of CADM1 in HepG2 cell line, Western blotting assay was performed. Anti-CADM1 (Abcam) and anti- $\beta$-actin (Cell Signaling) antibodies were used as primary antibodies, while horseradish peroxidase conjugated immunoglobulins were used as secondary antibodies. The results were visualized using enhanced chemiluminescence system (Amersham Pharmacia Biotech).

Proliferation assay. HepG2 cells seeded at a density of 5,000 cells per well into 96-well dishes were transfected with pcDNA3.1 (-)-CADM1 or empty pcDNA3.1 (-) vector using Lipofectamine 2000 (Invitrogen). After culturing the cells at the indicated time points $(0,1,3,5$ and $7 \mathrm{~d})$, a cell proliferation assay was done using Cell Counting Kit-8 (Dojindo) according to the manufacturer's protocol. Cell viability was expressed as percentage of optical density in pcDNA3.1 (-)-CADM1transfected cells compared with mock transfectants.

Combined bisulfite restriction analysis (COBRA). Based on the findings from the luciferase reporter assay, methylation status of $-240 /+39$ region including the basal promoter region of CADM1 was determined by COBRA in clinical HCC samples. Bisulfite-modified DNA was amplified with the primers which were same to the BSP primers to amplify $-240 /+39$ region in which four digestion sites of TaqI (New England Biolabs) were found. PCR products were digested with TaqI at $65^{\circ} \mathrm{C}$ for $1 \mathrm{~h}$, which recognizes sequences unique to methylated alleles but cannot recognize unmethylated alleles (19). Digested DNA fragments were visualized on $2.0 \%$ ethidium bromide-stained agarose gels.

Immunohistochemistry analysis. The expressions of CADM1 protein in 56 paired HCC tissues were detected by immunohistochemistry assay as described previously (20). Slides were incubated overnight at $4^{\circ} \mathrm{C}$ with rabbit anti-human polyclonal antibody against the C-terminus of CADM1 (1:400 dilution; TBS $/ 5 \%$ FBS $/ 0.1 \%$ Triton X-100; Abcam), and then stained with 3,3'-diaminobenzdine. Eventually, the slides were counterstained with hematoxylin, dehydrated and mounted. For negative control, primary antibody was replaced by rabbit immunoglobulin. Normal liver was taken as positive control. Semiquantitative evaluation was performed by two investigators independently, who were blind to clinical data. To compare the expression of CADM1 in neoplastic and adjacent non-neoplastc tissue, a proportion score and an intensity score were assigned to one sample, then added to obtain a total score according to the previous study (21).

Statistical analysis. The Chi-square test was used to determine the association between epigenetic alterations and different clinicopathologic features. DFS was measured from the operation day to the recurrent day or the most recent follow-up visit before May 31, 2010. DFS rates were assessed using KaplanMeier with a log-rank test to detect the statistical differences, and Cox proportional hazard model was performed for multivariate analysis of prognostic factors. Statistical analyses were carried out with SPSS 16.0 (SPSS, Inc., Chicago, IL). P<0.05 was considered statistically significant. 
A

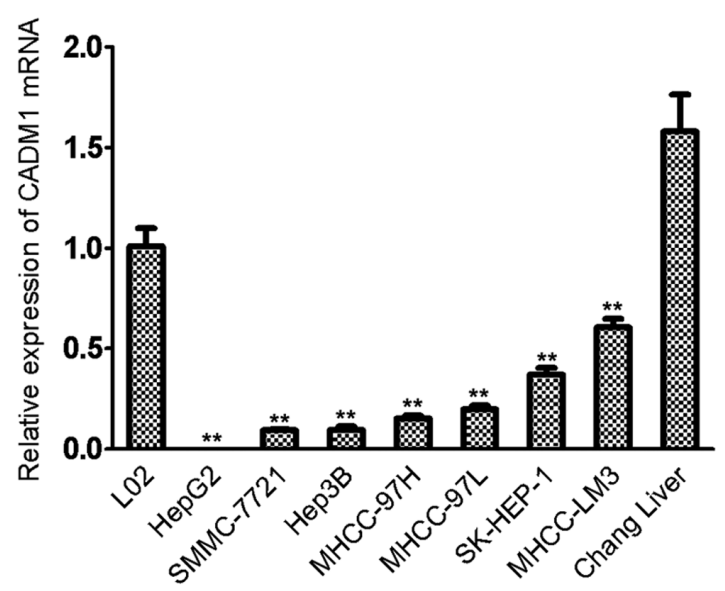

B

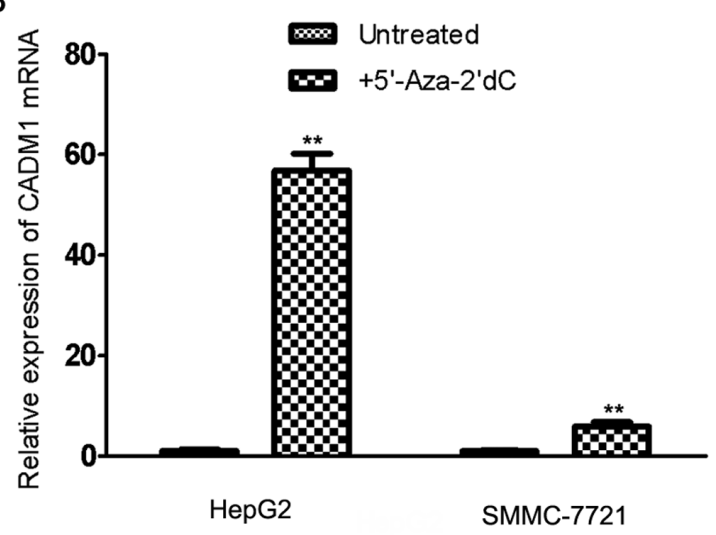

C

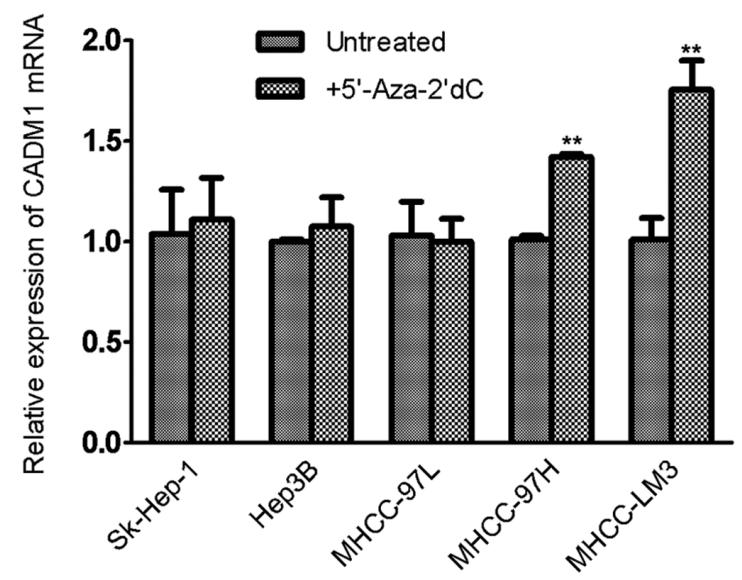

Figure 1. Transcriptive level of CADM1 in HCC cell lines. (A) Typical qRT-PCR results of mRNA expression levels of CADM1 in HCC cell lines compared with normal liver cell line L02. (B and C) The expression of CADM1 mRNA in HCC cell lines before and after 5-Aza-dC treatment Three independent experiments were done. ${ }^{*} \mathrm{P}<0.05 ;{ }^{* *} \mathrm{P}<0.01$.

\section{Results}

Gene silencing and methylation patterns of CADM1 promoter in HCC cell lines. To evaluate the potential of CADM1 as a tumor suppressor in HCC tumorigenesis, the mRNA expres- sion level of CADM1 in $7 \mathrm{HCC}$ cell lines were detected by qRT-PCR. Expression of CADM1 mRNA was decreased in all HCC cell lines compared with that in normal liver cell line L02 (Fig. 1A). To investigate the role of promoter methylation in silencing of CADM1 in HCC, we treated all HCC cell lines with 5-Aza-dC. As shown in Fig. 1B and C, up-regulated mRNA expression levels of CADM1 were found in SMMC7721, HepG2, MHCC-97H and MHCC-LM3, which indicates that DNA methylation of CADM1 promoter may contribute to the decreased CADM1 expression in certain HCC cells.

To further verify the relationship between the methylation status in the CADM1 promoter region and CADM1 expression level, the methylation status of CADM1 promoter was analyzed by BSP. Firstly, we analyzed the $\mathrm{CpG}$ island in CADM1 gene using MethPrimer software (22). One typical $\mathrm{CpG}$ island was found which located in the region of -652 to +252. The methylation status of $35 \mathrm{CpG}$ sites located in the promoter region of CADM1 ranging from -429 to +39 were detected. As shown in Fig. 2A, abberant hypermethylation of the CADM1 promoter was observed in 4 of $7 \mathrm{HCC}$ cell lines (57.1\%), in which CADM1 mRNA was decreased. However, CADM1 methylation was not detected in normal liver tissue nor in two immortalized human liver cell lines in which the expression level of CADM1 mRNA was high (Fig. 2A). We also investigated the methylation status in CADM1 promoter region in HepG2 and SMMC-7721 cell lines by BSP analysis after 5-Aza-dC treatment. The level of methylation was significantly decreased accompanied with up-regulation of CADM1 expression (Fig. 2B). These results further demonstrate that promoter methylation directly contributes to the silencing of CADM1 in certain HCC cells.

Analysis of CADM1 gene promoter region. To determine regulatory cis-elements that might be critical for expression of CADM1, 5'-serial deletion constructs consisting of CADM1 promoter was linked to luciferase reporter gene. As previous study has shown higher expression of CADM1 in the normal liver cell line (Chang Liver) using qRT-PCR analysis, we selected this cell line for transient transfection. The largest construct pGL4.17-1599/+234 as well as three other truncated constructs pGL4.17-635/+234, pGL4.17-364/+234 and pGL4.17-226/+234 displayed almost a similar level of $>20$-fold promoter activity over the empty pGL4.17 vector; however, there was a drastic decrease of promoter activity of $>75 \%$ with the truncated construct pGL4.17-146/+234 (Fig. 3A). These observations suggest that the fragment between -226 to -146 contains elements that confer high level of expression of the CADM1 gene in Chang Liver cells. Thus, the most critical region for the basal promoter activity of CADM1 gene is located within the $-226 /-146$ region. As shown in Fig. 2, abberant hypermethylation of this region was observed accompanied with decreased mRNA expression of CADM1 in HCC cells.

One $\mathrm{Spl}$ binding site was found in the proximal promoter region -226/-146 through searching for the Genomatix database. To verify the role of Sp1 in the regulation of CADM1 transcription, site-directed mutation of Spl site was introduced into the construct pGL4.17-226/+234. As shown in Fig. 3B and $\mathrm{C}$, mutation of the $\mathrm{Sp} 1$ binding site resulted in $70 \%$ reduction in the promoter activity compared to the wild-type 
A

5' Region of CADM1

TSS

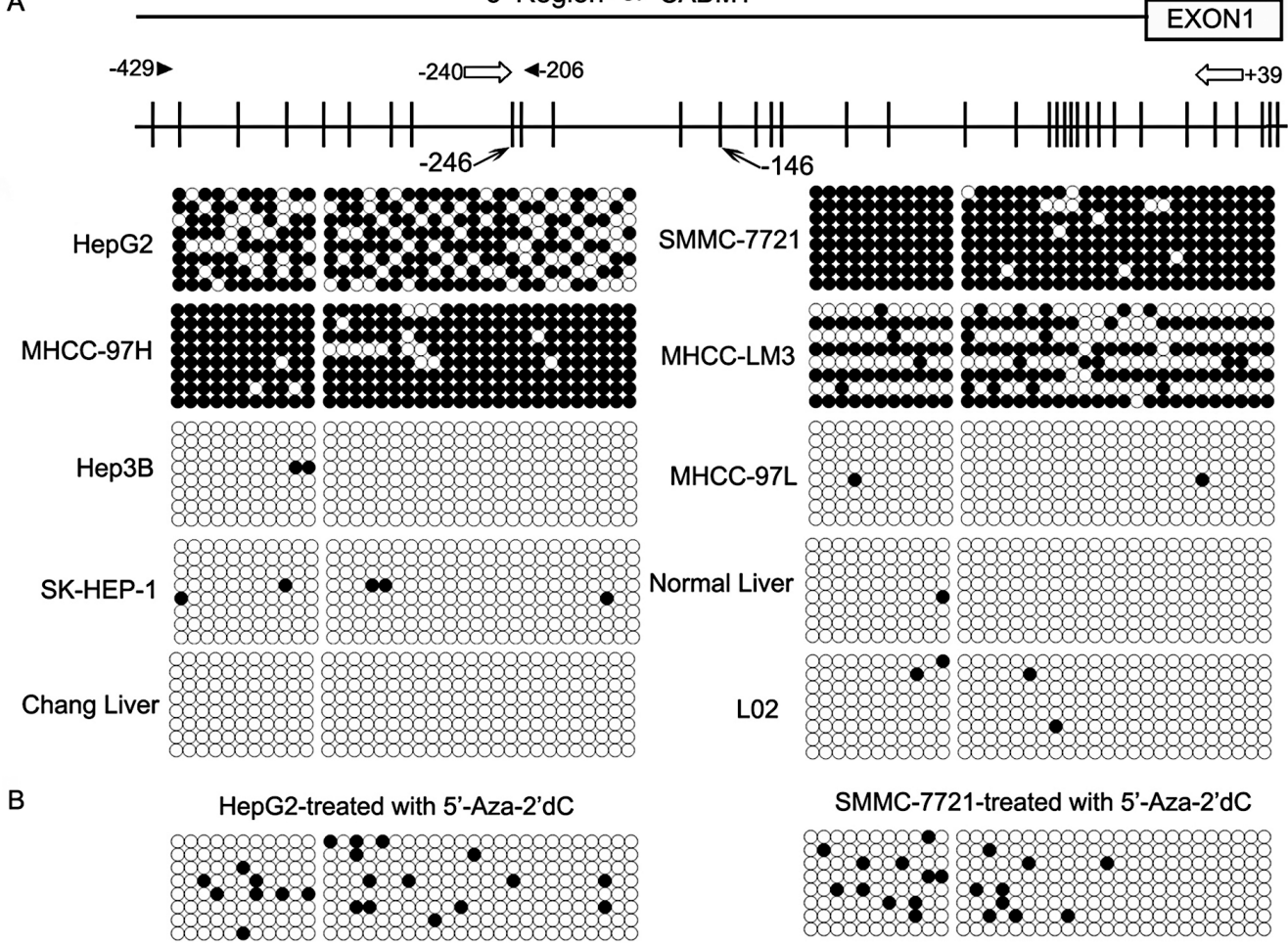

Figure 2. Methylation analysis of CADM1 promoter in HCC cell lines. (A) The results of sequence analysis in CADM1 promoter region. The primer pairs for bisulfite sequencing are shown (arrowheads). (B) Sequence analysis of CADM1 promoter after 5-Aza-dC treatment. Open circles, unmethylated cytosines; black circles, methylated sites in $\mathrm{CpG}$ sites.

A

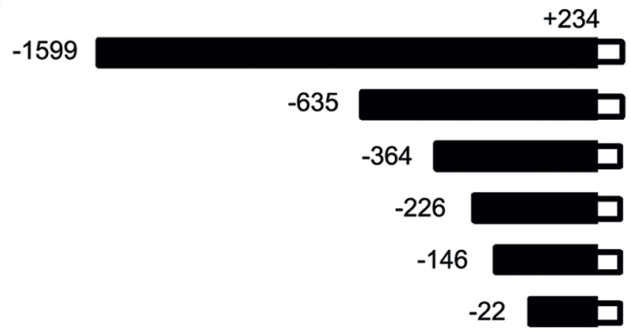

$P(-1599 /+234)$

$(-635 /+234)$

$P(-364 /+234)$

$(-226 /+234)$

$P(-146 /+234)$

$(-22 /+234)$

$\square$ PGL4.17control

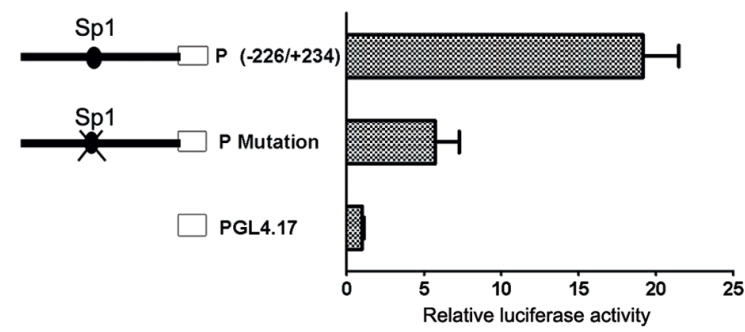

C

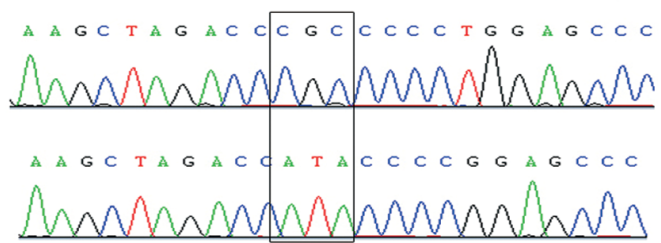

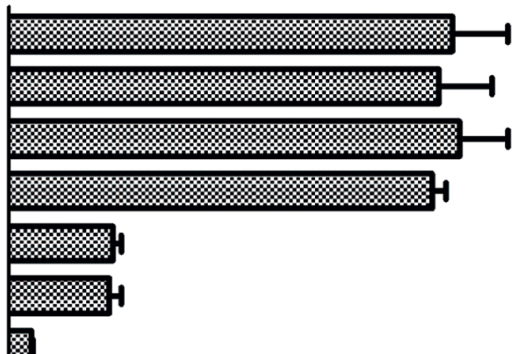

0

D

$-429$

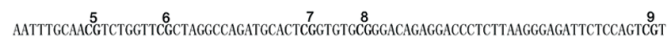
10.

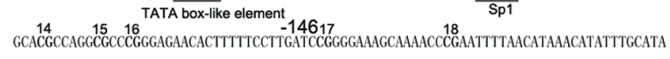

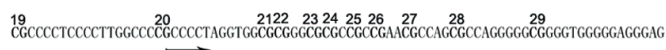

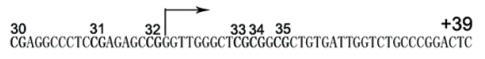

Figure 3. Functional analysis of the CADM1 promoter. (A) Left panel: various truncated constructs used in transient transfection luciferase assay. Right panel: luciferase activities in RLU (relative luciferase unit) of different truncated constructs in Chang Liver cells. The empty PGL4.17 vector was used as a negative control. The transcriptional start site is indicated as +1 . (B) Identification of Sp1 site important for CADM1 promoter activity. PGL4.17 (-226/+234) and PGL4.17 (-226/+234) mutant with Sp1 binding site mutation was transfected into Chang Liver cells, respectively. The luciferase activities were analyzed $48 \mathrm{~h}$ after transfection. (C) The sequencing results of Sp1 site mutation. Square frame was used to indicate the location of Sp1 site mutation. (D) Sequence analysis of CADM1 promoter region $(-429$ to +39$)$. CpG dinucleotides are numbered and shown in bold. One TATA box-like element and one predicated binding site for $\mathrm{Sp1}$ are underlined. Arrow, transcriptional start site. 
A

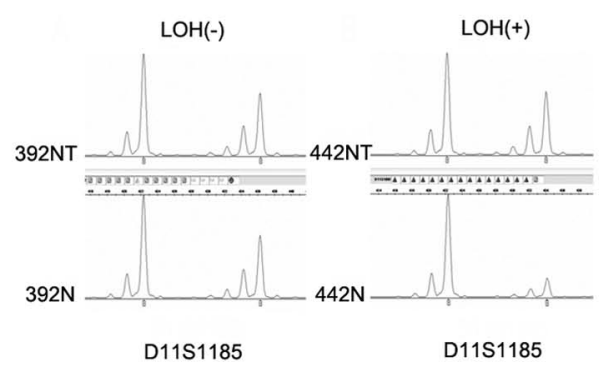

B

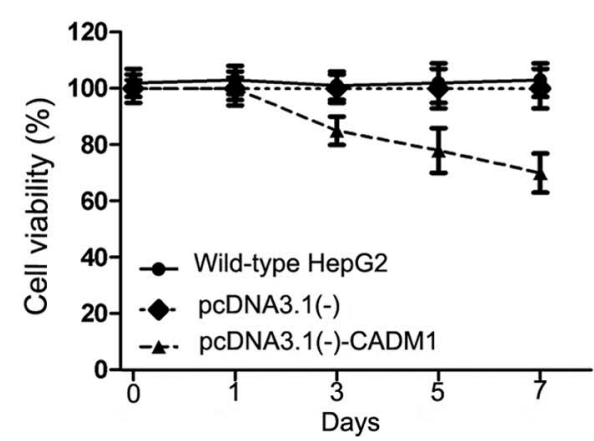

C

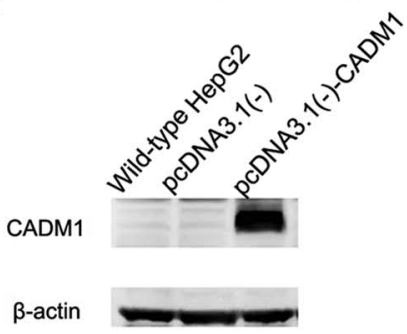

Figure 4. LOH analysis of CADM1 gene in clinical samples and anti-proliferation effects of CADM1 in vitro. (A) Representative examples of the genotyping profile at the D11S1185 locus showing LOH (+) and LOH (-) in HCC tissue samples, respectively. (B) Ectopic transfection of pcDNA3.1(-)-CADM1 in HepG2 cells resulted in a consistent diminution on cell viability relative to mock transfectants. Evaluation was based on means from six replicates in three independent experiments. ${ }^{*} \mathrm{P}<0.05$ compared with mock transfectants. (C) Identification of ectopic CADM1 expression by Western blot analysis.

A

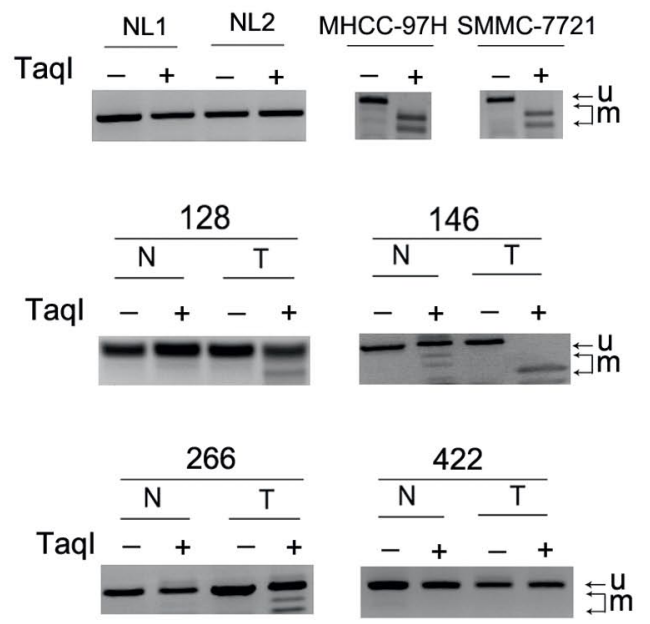

B
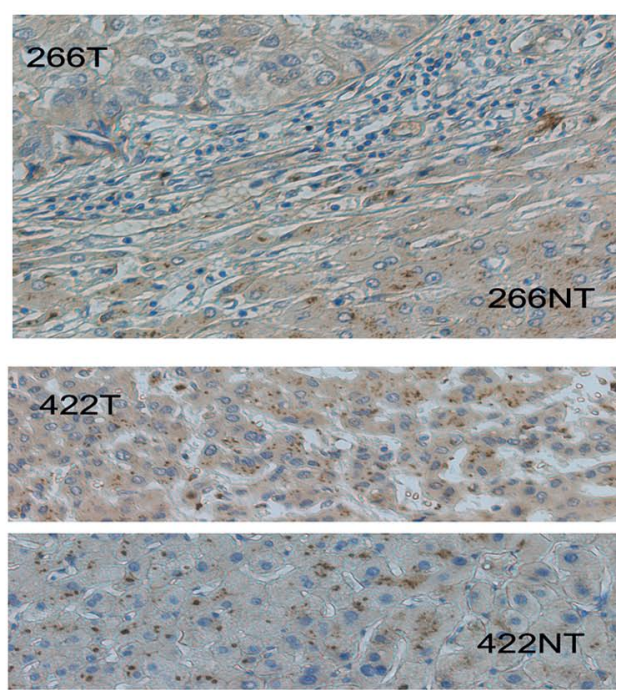

Figure 5. Analysis of CADM1 expression and methylation status in HCC tissue samples. (A) Results of COBRA analysis. Normal liver tissues (NL) was used as negative control; MHCC-97H and SMMC-7721 served as positive control. Arrows indicate unmethylated (u)/methylated (m) alleles. T, tumor samples; NT, adjacent non-tumor samples. (B) Immunohistochemical staining results for CADM1. Top, a tumor with methylated CADM1 showing negative CADM1 staining compared with positive CADM1 staining in the adjacent non-tumor tissue. Bottom, an unmethylated CADM1 tumor showing positive CADM1 staining both in tumor and the adjacent non-tumor tissue. Magnification, x200.

construct pGL4.17-226/+234, suggesting that Sp1 consensus motif was important for mediating basal CADM1 promoter activity. A TATA box-like element (TATAAACA) was also found ranging from -245 to -238 relative to the putative TSS in CADM1 gene (Fig. 3D). The results of luciferase report assay revealed that there was no difference of promoter activity between the construct pGL4.17-364/+234 contained the TATA box-like element and the construct pGL4.17-226/+234 without it (Fig. 3A).

LOH at CADM1 gene locus in HCC tissues. To explore other potential molecular mechanism of the down-regulation of CADM1, genomic imbalance of the CADM1 locus was investigated in 32 pairs of $\mathrm{HCC}$ specimens by $\mathrm{LOH}$ analysis. A representative genotyping profile of D11S1885 is shown in Fig. 4A. At the D11S1885 locus, 19 of 32 cases (59.4\%) were heterozygous and 2 of $19(10.5 \%)$ showed LOH. At the
D11S908 locus, 11 of 32 cases (34.4\%) were heterozygous and 3 of $11(27.3 \%)$ showed LOH. The heterozygosity of the D11S1992 locus was 20 of $32(62.5 \%)$, and 1 of $20(5.0 \%)$ exhibited LOH. In total, heterozygosity was detected in 29 of 32 cases $(90.6 \%)$, and $\mathrm{LOH}$ at the CADM1 locus was achieved in 4 of 29 cases $(13.8 \%)$. Of the 4 cases with $\mathrm{LOH}$ at the CADM1 locus, only one also showed CADM1 methylation. CADM1 protein expression was not detected in all of the 4 cases, so LOH might be one of the mechanisms of CADM1 gene silencing.

Ectopic CADM1 protein inhibited cell growth in HepG2 cells. The apparently high frequency of CADM1 silencing in HCC cell lines suggests that CADM1 may serve as a TSG in HCC. To test the potential tumor suppressor role of CADM1 in HCC, we introduced ectopic CADM1 protein into HepG2 cells to investigate whether CADM1 could suppress growth 
Table II. Characteristics of the patients and distribution of data in methylated cases and unmethylated cases.

\begin{tabular}{|c|c|c|c|c|}
\hline \multirow[b]{2}{*}{ Tumor parameters } & \multirow[b]{2}{*}{ Grading } & \multicolumn{3}{|c|}{ CADM1 methylation } \\
\hline & & Unmethylated, n (\%) & Methylated, n (\%) & aP-value \\
\hline Age (years) & $\begin{array}{l}\leq 50 \\
>50\end{array}$ & $\begin{array}{l}20(66.7) \\
28(54.2)\end{array}$ & $\begin{array}{l}10(33.3) \\
24(45.8)\end{array}$ & 0.256 \\
\hline Gender & $\begin{array}{l}\text { Female } \\
\text { Male }\end{array}$ & $\begin{array}{r}4(57.1) \\
44(59.1)\end{array}$ & $\begin{array}{r}3(42.9) \\
31(40.9)\end{array}$ & 1.000 \\
\hline Preoperative AFP level (ng/ml) & $\begin{array}{l}\leq 400 \\
>400\end{array}$ & $\begin{array}{l}25(71.4) \\
23(48.9)\end{array}$ & $\begin{array}{l}10(28.6) \\
24(51.1)\end{array}$ & 0.041 \\
\hline Tumor size $(\mathrm{cm})$ & $\begin{array}{l}\leq 5 \mathrm{~cm} \\
>5 \mathrm{~cm}\end{array}$ & $\begin{array}{l}30(60.0) \\
18(56.2)\end{array}$ & $\begin{array}{l}20(40.0) \\
14(43.8)\end{array}$ & 0.737 \\
\hline Tumor number & $\begin{array}{l}\text { Single } \\
\text { Multiple }\end{array}$ & $\begin{array}{l}28(60.9) \\
20(55.6)\end{array}$ & $\begin{array}{l}18(39.1) \\
16(44.4)\end{array}$ & 0.628 \\
\hline Histopathologic grading & $\begin{array}{c}\text { Well/moderate } \\
\text { Poor }\end{array}$ & $\begin{array}{l}32(62.7) \\
16(51.6)\end{array}$ & $\begin{array}{l}19(37.3) \\
15(48.4)\end{array}$ & 0.321 \\
\hline PVTT & $\begin{array}{l}\text { Negative } \\
\text { Positive }\end{array}$ & $\begin{array}{l}38(67.9) \\
10(38.5)\end{array}$ & $\begin{array}{l}18(32.1) \\
16(61.5)\end{array}$ & 0.012 \\
\hline Tumor recurrence & $\begin{array}{c}\text { Recurrence free } \\
\text { Recurrence }\end{array}$ & $\begin{array}{l}32(76.2) \\
16(40.0)\end{array}$ & $\begin{array}{l}10(23.8) \\
24(60.0)\end{array}$ & 0.001 \\
\hline
\end{tabular}

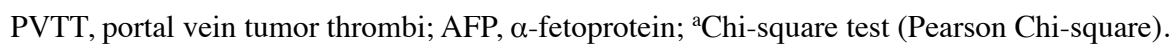

in HCC cell lines. As shown in Fig. 4B, a growth inhibitory effect was observed in HepG2 cell line. The difference was significant on the 3rd day. On the 7th day, results showed a nearly 30\% decrease in cell proliferation. Ectopic CADM1 expression was confirmed by Western blotting (Fig. 4C).

CADM1 promoter methylation and CADM1 expression in primary $H C C$. To ascertain whether the aberrant hypermethylation of CADM1 promoter in HCC cell lines could be detected in primary HCC tissues, the methylation status of CADM1 promoter was determined in all $82 \mathrm{HCC}$ tissues (including 56 paired samples). Aberrant hypermethylation of CADM1 was detected in 34 of $82(41.5 \%)$ of HCC tissues (Fig. 5A). In 56 paired HCC tissues, aberrant hypermethylation was observed in 9 of $56(16.1 \%)$ of adjacent non-tumor tissues and 23 of $56(41.1 \%)$ of HCC tissues. The detection of methylation in a small number of adjacent non-tumor tissues samples might be explained by the occurrence of methylation in preneoplastic/preinvasive versions. However, CADM1 promoter methylation was not detected in any of the 8 normal liver tissues (Fig. 5A).

Immunohistochemistry was used to investigate the relationship between aberrant methylation status and expression patterns of CADM1 in 56 paired HCC tissue samples. Representative immunohistochemical images of CADM1 expression are shown in Fig. 5B. Aberrant methylation of CADM1 was observed in 20 of 39 (51.3\%) of HCC tissues with CADM1 immunostaining negative, which was much higher than that in CADM1 immunostaining positive HCC tissues ( 3 of 17, $17.6 \%$ ). Then, a statistically significant correlation between promoter methylation and down-regulation of CADM1 was established in vivo $(\mathrm{P}=0.019)$. However, the underexpression of CADM1 without promoter methylation in some HCC cases might be attributed to other mechanisms such as $\mathrm{LOH}$, and/or other epigenetic alterations.

Aberrant methylation status of CADM1 promoter is associated with poor prognosis in HCC patients treated with LT. The relationship of CADM1 methylation with the clinicopathologic parameters was analyzed in $82 \mathrm{HCC}$ cases. A higher recurrence was observed in patients with aberrant methylation of CADM1 promoter than patients without CADM1 methylation (70.6\% versus $33.3 \%$; $\mathrm{P}=0.001)$. Notably, aberrant methylation of CADM1 promoter was more frequent in HCC cases with high serum level of $\alpha$-fetoprotein (AFP) $>400 \mathrm{ng} / \mathrm{ml}$ than those with low serum level of AFP $\leq 400 \mathrm{ng} / \mathrm{ml}$ (70.6\% versus $47.9 \%$; $\mathrm{P}=0.041$ ). Furthermore, our results showed that aberrant methylation of CADM1 positively correlated with portal vein tumor thrombi (PVTT) $(\mathrm{P}=0.012)$. No statistically significant correlations were found between CADM1 methylation status and other clinicopathological findings including age, gender, tumor number, tumor size and histopathologic grading (Table II).

We were able to follow up with all 82 patients originally involved in this study. Complete follow-up data were obtained, and the follow-up time ranged from 2 to 68 months. Using Kaplan-Meier method and log-rank test, the results showed that the mean DFS for CADM1-unmethylated patients was significantly longer than that for CADM1-methylated patients (32.5 versus 15.2 months, $\mathrm{P}=0.001$, Fig. 6). DFS of patients was also positively correlated with the clinicopathologic findings including AFP, PVTT, tumor size and histopathologic 
Table III. Prognostic value of CADM1 methylation and clinicopathological characteristics for disease-free survival in 82 patients with HCC.

\begin{tabular}{|c|c|c|c|c|}
\hline \multirow[b]{2}{*}{ Variables } & \multirow{2}{*}{$\frac{\begin{array}{c}\text { Kaplan-Meier } \\
\text { log-rank test }\end{array}}{\text { P-value }}$} & \multicolumn{3}{|c|}{ Multivariate proportional hazards model } \\
\hline & & Hazards ratio & $95 \% \mathrm{CI}$ & $\mathrm{P}$-value \\
\hline Age & 0.528 & NS & & \\
\hline Gender & 0.225 & NS & & \\
\hline Tumor number & 0.567 & NS & & \\
\hline Grade & 0.040 & 1.563 & $1.201-3.175$ & 0.240 \\
\hline Size & 0.038 & 1.423 & $0.872-2.929$ & 0.266 \\
\hline PVTT & 0.015 & 1.695 & $0.996-3.324$ & 0.062 \\
\hline AFP & 0.006 & 2.065 & $1.020-3.409$ & 0.016 \\
\hline CADM1 methylation & 0.001 & 2.788 & $1.043-5.063$ & 0.010 \\
\hline
\end{tabular}

95\% CI, 95\% confidence interval; NS, not selected in multivariate proportional hazards model.

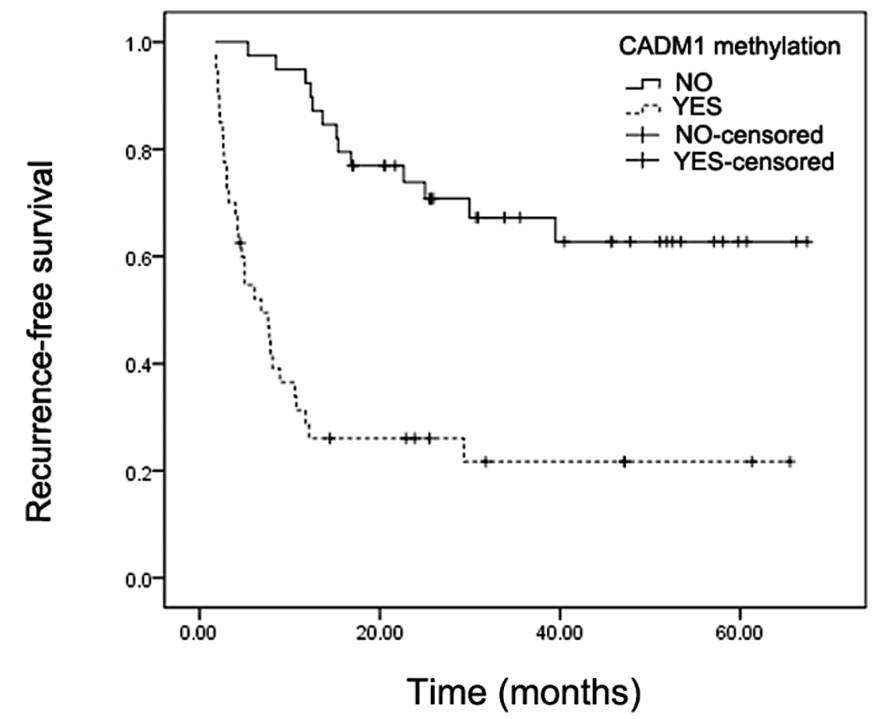

Figure 6. Kaplan-Meier estimates of cumulative recurrence-free survival in the HCC patients treated with liver transplantation according to the methylation profile.

grading (Table III). These five clinicopathologic factors found to be prognostic on univariate analysis were entered into a multivariate analysis to identify independent predictors of DFS. Multivariate analysis using the Cox proportional hazards model revealed that CADM1 methylation status $(\mathrm{HR}=2.788$; 95\% CI, 1.043-5.063; $\mathrm{P}=0.010)$ and serum level of AFP $(\mathrm{HR}=2.065 ; 95 \% \mathrm{CI}, 1.020-3.409 ; \mathrm{P}=0.016)$ were independent prognostic factors for DFS of HCC patients treated with LT.

\section{Discussion}

In the present study, we described that CADM1 was frequently inactivated by promoter hypermethylation in HCC, both in vivo and in vitro. $\mathrm{LOH}$ was another possible mecha- nism for transcriptional regulation. We also identified the proximal promoter region of CADM1 gene for the first time. Furthermore, the methylation status of CADM1 was associated with the clinicopathologic parameters and recurrence of HCC. To our best knowledge, this is the first study to investigate the relationship between the methylation status of CADM1 and the clinicopathologic parameters in HCC patients.

CADM1 was initially identified as a tumor suppressor gene by its suppressor activity of tumor formation in BALB/c athymic Foxn1 nu/nu mice (9). In the present experiment, ectopic CADM1 expression in HepG2 cells resulted in suppressed cell growth supporting the notion that CADM1 may function as a TSG in HCC. Silencing of CADM1 by aberrant hypermethylation has been reported in various cancers, including NSCLC, nasopharyngeal carcinoma, breast cancer, esophageal carcinoma and cervical cancer (9,14,23-26). In our study, a relatively large number of $35 \mathrm{CpG}$ sites was evaluated to get more detailed methylation profile of CADM1 promoter in HCC cells (Fig. 2). The detailed analysis undertaken has established a good correlation between CADM1 silencing and aberrant hypermethylation in HCC. Notwithstanding contributions by other mechanisms, such as LOH (13.8\%), our data indicate that promoter methylation is a major mechanism for silencing of CADM1 during HCC carcinogenesis.

To further explore the potential mechanism relevant to the transcriptional regulation of CADM1 in HCC, the most critical region for the basal promoter activity of CADM1 gene has been identified. Previous studies revealed that Sp1 was a critical factor in the transcriptional regulation $(27,28)$. Our results of site-directed mutation assay suggested that the consensus Sp1 binding site plays the primary role within the CADM1 promoter (Fig. 3). Recently, some reports demonstrated that methylation of the Sp1 consensus motifs and adjacent $\mathrm{CpG}$ sites might block Sp1 binding and transactivation $(29,30)$. Thus, hypermethylation of Sp1 binding site and adjacent $\mathrm{CpG}$ sites may directly reduce $\mathrm{Sp} 1$ recruitment, therefore leading to a decreased CADM1 expression in HCC. TATA box was 
once believed to be an essential component of core promoters, which is challenged by advanced studies (31). A consensus TATA element does not appear to be a major determinant of either TATA box binding protein binding or gene expression throughout the genome in yeast. Thus, TATA box may not be a critical factor for determining promoter activity even when it is found in a core promoter of some genes (31-33). In the present study, the results of luciferase report assay indicated that the TATA box-like element in CADM1 does not play a primary role for promoter activity. Considering the location of the TATA box-like element, it was far relative to the TSS, and there was a single mutation compared to the consensus sequence of TATA box $\operatorname{TATA}(\mathrm{A} / \mathrm{T}) \mathrm{A}(\mathrm{A} / \mathrm{T})(\mathrm{A} / \mathrm{G})(34)$; the TATA box-like element in the region of CADM1 promoter maybe not a bona fide TATA box.

The purpose of promoter region analysis was to acquire the most critical region for basal promoter activity of CADM1 gene, so then we can focus on this region to get a real status of methylation in the HCC clinical samples. In the present study, we confirmed that the DNA hypermethylation at core promoter and putative Sp1 binding site was wellcorrelated with the aberrant CADM1 expression in primary HCC samples. There is emerging evidence that methylation status of CADM1 is associated with clinicopathologic features and prognosis in various cancers $(12,14,24)$. Murakami et al (35) demonstrated that aberrant hypermethylation of CADM1 was significantly associated with a shorter DFS, providing an independent prognostic factor in the patients with lung adenocarcinoma. Our data demonstrate that methylation of CADM1 was significantly associated with AFP, PVTT and tumor recurrence in patients with HCC. Furthermore, CADM1 methylation provides an independent prognostic factor for DFS. Those findings raise the possibility that CADM1 methylation may serve as a hopeful biomarker for monitoring $\mathrm{HCC}$ recurrence.

In conclusion, our data revealed that epigenetic silencing of CADM1 through methylation is an important event in the pathogenesis of HCC. The prognostic potential of CADM1 biomarker may provide the physician with better knowledge on HCC recurrence in LT patients. More accurate risk prediction may help individualize follow-up strategies, e.g. the frequency of follow-up, or it may be an aid when interpreting increasing AFP. However, underlying mechanism of tumor suppression by CADM1 is not clarified yet in HCC, further investigation will be required.

\section{Acknowledgments}

This study was supported by the National S\&T Major Project (no. 2008ZX10002-026) and by the National Basic Research Program of China (973 program no. 2009CB522403).

\section{References}

1. Parkin DM, Bray F, Ferlay $\mathbf{J}$ and Pisani P: Global cancer statistics, 2002. CA Cancer J Clin 55: 74-108, 2005.

2. Thorgeirsson SS and Grisham JW: Molecular pathogenesis of human hepatocellular carcinoma. Nat Genet 31: 339-346, 2002.

3. Zimmerman MA, Ghobrial RM, Tong MJ, et al: Recurrence of hepatocellular carcinoma following liver transplantation: a review of preoperative and postoperative prognostic indicators. Arch Surg 143: 182-188, 2008
4. Baylin SB and Ohm JE: Epigenetic gene silencing in cancer - a mechanism for early oncogenic pathway addiction? Nat Rev Cancer 6: 107-116, 2006.

5. Esteller M: Epigenetics in cancer. N Engl J Med 358: 1148-1159, 2008.

6. Esteller M: Cancer epigenomics: DNA methylomes and histonemodification maps. Nat Rev Genet 8: 286-298, 2007.

7. Weiss G, Cottrell S, Distler J, et al: DNA methylation of the PITX2 gene promoter region is a strong independent prognostic marker of biochemical recurrence in patients with prostate cancer after radical prostatectomy. J Urol 181: 1678-1685, 2009.

8. Wemmert S, Bettscheider M, Alt S, et al: p15 promoter methylation - a novel prognostic marker in glioblastoma patients. Int $\mathrm{J}$ Oncol 34: 1743-1748, 2009.

9. Kuramochi M, Fukuhara H, Nobukuni T, et al: TSLC1 is a tumor-suppressor gene in human non-small-cell lung cancer. Nat Genet 27: 427-430, 2001.

10. Fu L, Gao Z, Zhang X, et al: Frequent concomitant epigenetic silencing of the stress-responsive tumor suppressor gene CADM1, and its interacting partner DAL-1 in nasal NK/T-cell lymphoma. Int J Cancer 124: 1572-1578, 2009.

11. Berginc $G$, Bracko $M$ and Glavac D: MS-MLPA reveals progressive age-dependent promoter methylation of tumor suppressor genes and possible role of IGSF4 gene in colorectal carcinogenesis of microsatellite instable tumors. Cancer Invest 28: 94-102, 2009.

12. Li J, Zhang Z, Bidder M, et al: IGSF4 promoter methylation and expression silencing in human cervical cancer. Gynecol Oncol 96: 150-158, 2005.

13. Murakami Y: Involvement of a cell adhesion molecule, TSLC1/ IGSF4, in human oncogenesis. Cancer Sci 96: 543-552, 2005.

14. Heller G, Fong KM, Girard L, et al: Expression and methylation pattern of TSLC1 cascade genes in lung carcinomas. Oncogene 25: 959-968, 2006.

15. Lung HL, Cheung AK, Xie D, et al: TSLC1 is a tumor suppressor gene associated with metastasis in nasopharyngeal carcinoma. Cancer Res 66: 9385-9392, 2006.

16. Zhang F, Wu LM, Zhou L, et al: Predictive value of expression and promoter hypermethylation of XAF1 in hepatitis B virusassociated hepatocellular carcinoma treated with transplantation. Ann Surg Oncol 15: 3494-3502, 2008.

17. Livak KJ and Schmittgen TD: Analysis of relative gene expression data using real-time quantitative PCR and the 2[-Delta Delta C(T)] method. Methods 25: 402-408, 2001.

18. Doldan A, Chandramouli A, Shanas R, et al: Loss of the eukaryotic initiation factor $3 \mathrm{f}$ in pancreatic cancer. Mol Carcinog 47: 235-244, 2008.

19. Xiong $Z$ and Laird PW: COBRA: a sensitive and quantitative DNA methylation assay. Nucleic Acids Res 25: 2532-2534, 1997.

20. Overmeer RM, Henken FE, Snijders PJ, et al: Association between dense CADM1 promoter methylation and reduced protein expression in high-grade CIN and cervical SCC. J Pathol 215: 388-397, 2008.

21. Lu B, Ma Y, Wu G, et al: Methylation of Tip30 promoter is associated with poor prognosis in human hepatocellular carcinoma. Clin Cancer Res 14: 7405-7412, 2008.

22. Li LC and Dahiya R: MethPrimer: designing primers for methylation PCRs. Bioinformatics 18: 1427-1431, 2002.

23. Hui AB, Lo KW, Kwong J, et al: Epigenetic inactivation of TSLC1 gene in nasopharyngeal carcinoma. Mol Carcinog 38: $170-178,2003$.

24. Heller G, Geradts J, Ziegler B, et al: Downregulation of TSLC1 and DAL-1 expression occurs frequently in breast cancer. Breast Cancer Res Treat 103: 283-291, 2007.

25. Ito T, Shimada Y, Hashimoto Y, et al: Involvement of TSLC1 in progression of esophageal squamous cell carcinoma. Cancer Res 63: 6320-6326, 2003.

26. Steenbergen RD, Kramer D, Braakhuis BJ, et al: TSLC1 gene silencing in cervical cancer cell lines and cervical neoplasia. J Natl Cancer Inst 96: 294-305, 2004.

27. Ali AM, Bajaj V, Gopinath KS and Kumar A: Characterization of the human SLC22A18 gene promoter and its regulation by the transcription factor Sp1. Gene 429: 37-43, 2009.

28. Wang J, Liu X, Ni P, Gu Z and Fan Q: SP1 is required for basal activation and chromatin accessibility of CD151 promoter in liver cancer cells. Biochem Biophys Res Commun 393: 291-296, 2010.

29. Zhu WG, Srinivasan K, Dai Z, et al: Methylation of adjacent CpG sites affects Sp1/Sp3 binding and activity in the p21(Cip1) promoter. Mol Cell Biol 23: 4056-4065, 2003. 
30. Guendel I, Carpio L, Pedati C, et al: Methylation of the tumor suppressor protein, BRCA1, influences its transcriptional cofactor function. PLoS One 5: e11379, 2010.

31. Muller F, Demeny MA and Tora L: New problems in RNA polymerase II transcription initiation: matching the diversity of core promoters with a variety of promoter recognition factors. J Biol Chem 282: 14685-14689, 2007.

32. Basehoar AD, Zanton SJ and Pugh BF: Identification and distinct regulation of yeast TATA box-containing genes. Cell 116: 699-709, 2004.

33. Kim J and Iyer VR: Global role of TATA box-binding protein recruitment to promoters in mediating gene expression profiles. Mol Cell Biol 24: 8104-8112, 2004.
34. Yang C, Bolotin E, Jiang T, Sladek FM and Martinez E: Prevalence of the initiator over the TATA box in human and yeast genes and identification of DNA motifs enriched in human TATA-less core promoters. Gene 389: 52-65, 2007.

35. Kikuchi S, Yamada D, Fukami T, et al: Hypermethylation of the TSLC1/IGSF4 promoter is associated with tobacco smoking and a poor prognosis in primary nonsmall cell lung carcinoma. Cancer 106: 1751-1758, 2006. 